ISSN 1782-2041

DOI 10.25518/1782-2041.1203

\title{
Husserl et la délimitation ontologique de la culture
}

\author{
PAR MARCUS SACRINI \\ Universidade de São Paulo \\ $\bowtie$ sacrini@usp.br
}

Résumé L'article reprend les descriptions husserliennes de la culture comme composant d'une région ontologique matérielle spécifique, celle de l'esprit. D'abord, le programme général d'ontologies matérielles est brièvement décrit. Ensuite, on montre que la culture est entremêlée à la socialité dans la région de l'esprit. Pour bien indiquer la spécificité des relations culturelles, on reconstruit la notion de prédicat de sens ainsi que les processus d'objectivation et de transmission des objectités déterminées par de tels prédicats.

Mots-clefs Husserl, culture, ontologie, tradition.

\section{Introduction}

On trouve des références à la culture dans de nombreux textes publiés et manuscrits posthumes de Husserl. Il n'est pas immédiatement évident que toutes ces références s'accordent en une réflexion systématique ou si elles introduisent des approches différentes. Des commentateurs se sont déjà consacrés à ce sujet et y ont apporté de nombreuses analyses contribuant à sa systématisation ${ }^{1}$. En ce qui nous concerne, nous souhaitons prendre part à cette discussion

\footnotetext{
${ }^{1}$ Deux œuvres récentes importantes à propos du rôle de la culture dans l'œuvre de Husserl sont : Perreau, L. Le monde social selon Husserl. New York : Springer, 2013 ; Caminada, E. Vom Gemeingeist zum Habitus : Husserls Ideen II. New York : Springer, 2019.
} 
en soulignant comment plusieurs des analyses husserliennes de la culture se sont organisées autour d'un projet commun, à savoir, la formulation de l'ontologie régionale de l'esprit. Tout d'abord, nous reprendrons le sens de la perspective ontologique chez Husserl et nous verrons de quelle manière l'auteur l'applique au thème de l'esprit. Ensuite, nous chercherons à retracer les composantes eidétiques de la culture en tant que partie de cette région d'être.

\section{Phénoménologie et ontologie}

Reprenons vite l'armature méthodologique développée par Husserl au moins depuis le «tournant» transcendantal de 1906. La phénoménologie transcendantale cherche à décrire la constitution du sens de l'expérience à partir des opérations du sujet compris comme pur, c'est-à-dire, indépendamment des circonstances réelles particulières. On prétend, au moyen de cette analyse, expliciter ce qui, en dernière instance, rend l'expérience significative, à savoir des capacités intentionnelles qui permettent, chacune à son niveau spécifique, des manifestations objectivantes. Selon Husserl, pour faire progresser cette recherche, il est nécessaire de rompre avec des habitudes de pensée profondément enracinées, qui accentuent dans chaque expérience les objets, et qui ne s'intéressent guère à l'apparaître même. Le point méthodologique central de la recherche phénoménologique consistera, alors, à suspendre la validité de la position d'être contenue dans toute expérience naturelle ${ }^{1}$, afin de rendre explicites les sources subjectives qui constituent le sens de cette notion même d'être. Au moyen de la réduction phénoménologique, on inhibe l'intérêt pour l'être de ce qui apparaît, et on décrit le simple apparaître phénoménal de tout ce que la conscience appréhende. Ainsi, on cherche à rendre visibles les opérations intentionnelles qui, selon des légalités a priori, constituent, sur la base d'une phénoménalité pure et absolument évidente, le sens d'être objectif attribué aux choses et au monde dans l'expérience naturelle. C'est au moyen de cette modification méthodique de l'expérience naturelle que Husserl dévoile « le sol de

\footnotetext{
${ }^{1}$ Selon Husserl, le monde et tout ce qui le compose sont habituellement donnés à la conscience de manière effective, comme effectivement existant, et cette donation signifie que son être effectif apparaît comme constaté, en d'autres termes, imposé à la conscience. (Cf. Idées directrices pour une phénoménologie pure et une philosophie phénoménologique. Trad. de J. F. Lavigne. Paris : Gallimard, 2018, §30 ; cité désormais IdI). Par conséquent, il prévaut, dans l'expérience naturelle, une thèse générale d'être, laquelle sera mise hors circuit par la réduction phénoménologique.
} 
l'expérience transcendantale ${ }^{1}$, c'est-à-dire, le noyau phénoménal évident à partir duquel tout le sens d'être présent dans les expériences vécues quotidiennes est constitué.

Notons que ce schéma méthodologique admet l'antériorité de l'expérience mondaine. Les opérations transcendantales seront révélées sur la base des données de l'expérience naturelle, qui sert de guide pour l'éclaircissement des opérations de la subjectivité. En un mot, Husserl considère l'expérience naturelle comme le fil conducteur pour les analyses constitutives transcendantales, et c'est à travers cette fonction, comme nous le verrons plus loin, que le thème de la culture sera examiné. On part de la donation des objets pour, ensuite, de façon régressive, rendre apparent le système d'actes subjectifs qui attribuent le sens d'être à ce qui apparaît. La phénoménologie cherche donc à révéler la structure a priori de la subjectivité, non pas comme une sorte d'étant autonome isolé, mais comme intrinsèquement liée à différents types de pôles objectifs.

Il convient de souligner que Husserl attribue le rôle de fils conducteurs à des thèmes objectifs considérés comme ontologiquement autonomes, c'està-dire non encore réduits transcendantalement. Il existe en fait dans différents textes une longue exploration des caractéristiques constituantes des domaines spécifiques, qui offriront des voies spécifiques de passage pour la réflexion transcendantale ${ }^{2}$. Cette description systématique des domaines objectifs selon leurs caractéristiques générales, encore sans préoccupations transcendantales, marque pour Husserl le développement des disciplines scientifiques ontologiques. Au moins depuis Idées I, Husserl propose que des ontologies soient élaborées dans le but de contribuer à l'explication des fondements du savoir scientifique $^{3}$. Dans cette œuvre, le phénoménologue fait la distinction entre connaissance formelle et connaissance matérielle. La première est dérivée des concepts logiques ou mathématiques et se caractérise par sa validité formelle, puisqu'elle n'est pas restreinte par des particularités thématiques et peut être appliquée aux plus différents domaines du savoir. À son tour, la connaissance matérielle se réfère à des genres objectifs qui délimitent des régions d'être. Il importe de souligner que la connaissance matérielle n'est pas nécessairement

${ }^{1}$ Husserl, E. «Phänomenologie und Anthropologie», dans Aufsätze und Vorträge. 1922-1937. Hua XXVII. Den Haag: Kluwer, 1988, p. 172.

${ }^{2}$ Cette manière de procéder est thématisée par Husserl dans Idées $I$ : « La hiérarchie des eidétiques formelles et matérielles préfigurent, d'une certaine manière, la hiérarchie des phénoménologies constitutives, détermine leurs niveaux de généralité et leur donne leurs « fils conducteurs » dans les concepts fondamentaux et les propositions fondamentales des ontologies et des eidétiques matérielles. » (IdI, § 153, p. 455-456).

${ }^{3}$ Cf. IdI, $\S \S 7-9,16$. 
empirique ou factuelle, mais peut être explorée eidétiquement, c'est-à-dire, avec une validité a priori, bien que sans l'universalité vide de la connaissance formelle. Husserl estime que n'importe quel objet ou événement matériel singulier peut être étudié à partir de ses caractéristiques définitionnelles génériques, ce qui rend visibles certaines déterminations invariantes qui justifient de classer cet objet comme d'un type et non d'un autre. Il est alors possible d'analyser les caractéristiques matérielles dans des degrés de généralité de façon à rendre explicites des essences matérielles. Par conséquent, les événements empiriques acceptent un type de recherche qui ne se limite pas à recenser des cas concrets, mais qui cherche à tracer les caractéristiques eidétiques instanciées dans chaque exemplaire du thème traité. Les caractéristiques définitionnelles des types matériels peuvent être hiérarchisées en degrés de généralité jusqu'à ce que se révèlent les genres suprêmes de l'être matériel. Husserl suggère le développement de disciplines spécifiques qui systématiseraient la connaissance eidétique de chaque région matérielle, à savoir, les ontologies régionales. Ces disciplines fourniraient les délimitations a priori pour chaque type d'être mondain, ce qui permettrait l'anticipation dans les phénomènes empiriques (selon qu'ils appartiennent à un ou autre type de région) de certaines caractéristiques définitionnelles du genre matériel suprême qu'ils instancient ${ }^{1}$.

Revenons à présent aux genres suprêmes matériels. Quelles sont, selon Husserl, les ultimes régions ontologiques qui délimitent les types d'êtres mondains ? Un genre suprême est celui de la nature, lequel comprend, pour sa part, une subdivision bipartite. Husserl mentionne les domaines des choses physiques et de la nature animée comme les principales sous-régions eidétiques, ordonnées de manière stratifiée, c'est-à-dire, par des couches qui se superposent en niveaux de plus en plus complexes. La nature physique serait la couche fondatrice sur laquelle s'ordonnerait la nature animée ou organique, laquelle

\footnotetext{
${ }^{1}$ Au paragraphe 153 de Idées I, Husserl précise : « Le phénoménologue ne forme pas un jugement ontologique lorsqu'il reconnaît dans un concept ou une proposition ontologique l'index de connexions eidétiques constitutives, lorsqu'il y voit un fil conducteur menant à des mises en évidences intuitives qui portent purement en elles-mêmes leur légitimité et leur valeur ». (IdI, p. 455-456). Ainsi, le phénoménologue utilise les concepts constitutifs des ontologies sous forme d'indices qui renvoient à un ensemble d'opérations subjectives responsables de leur validation. Il revient au phénoménologue d'étudier ces opérations, lesquelles se révèlent plus facilement en tant qu'elles sont reliées à des disciplines ontologiques correspondantes. Le développement des ontologies n'est pas une tâche qui se réalise au sein de la réduction transcendantale, mais plutôt une investigation théorique pré-phénoménologique qui offre un fil conducteur à la phénoménologie.
} 
dépend de la base physique pour s'organiser, bien qu'elle présente des caractéristiques propres qu'on ne rencontre pas dans le niveau précédent. La sphère des événements psychiques marquerait une subdivision à l'intérieur de la nature animée, mais également fondée dans les niveaux précédents ${ }^{1}$. Au-delà de de ce grand domaine ontologique, Husserl reconnaît la région de la conscience pure comme un autre genre suprême. Cela veut dire que certaines formes de structurations phénoménales renvoient exclusivement à des restrictions $a$ priori provenant de l'être conscience lui-même, lequel ne se réduit pas à l'ordonnancement psychosomatique naturel, bien que celui-là soit partiellement fondé sur celui-ci² ${ }^{2}$.

Notons maintenant que Husserl admet une troisième région d'être matériel, c'est-à-dire, un troisième type d'ordonnancement phénoménal basé sur des déterminations matérielles a priori non réductibles aux ordres d'être reconnus précédemment, à savoir, nature et conscience. Il s'agit du domaine de l'esprit. Les « communautés intersubjectives », par exemple, font partie de ce domaine. Husserl affirme :

Ces communautés, quoique essentiellement fondées sur des réalités psychiques naturelles, qui sont pour leur part fondées sur des réalités physiques, se révèlent être des objectités d'ordre supérieur d'un type nouveau. Il s'avère, en général, qu'il existe de nombreux types d'objectités qui défient toutes les réinterprétations psychologistes et naturalistes. Ainsi de tous les objets axiologiques et objets pratiques, de toutes les formations culturelles concrètes qui, telles de dures réalités, déterminent notre vie effective, par exemple l'État, le droit, les moeurs, l'Eglise, etc. Toutes ces objectités doivent être décrites comme elles parviennent à l'état de donné, en fonction de leurs types fondamentaux et selon l'ordre de leur stratification, et il faut poser et résoudre à leur propos les problèmes de la constitution ${ }^{3}$.

Husserl indique ici l'autonomie ontologique des relations interpersonnelles et des objets et institutions culturelles, sans pour autant rejeter sa fondation dans

${ }^{1}$ Cf. Husserl, E. Ideen zur einer reinen Phänomenologie und phänomenologischen Philosophie. Zweites Buch: Phänomenologische Untersuchungen zur Konstitution. Hua IV. Den Haag : Martinus Nijhoff, 1952, §§ 20, 30.

${ }^{2}$ Conforme cité dans la note antérieure, Husserl défend, dans Idées II, que les phénomènes psychiques sont eidétiquement liés à la base corporelle. Il est intéressant de contraster cette analyse avec celle du paragraphe 54 de Idées I, où est évoquée la possibilité de la conscience sans âme, c'est-à-dire, sans des connexions avec le corps. Cette possibilité a du sens exactement à la lumière du projet de discerner les caractéristiques de la conscience pure en tant que source dernière du sens.

${ }^{3}$ IdI, $\S 152$, p. 449-450. 
les couches de la conscience et de la nature ${ }^{1}$. En outre, il insiste sur la nécessité de décrire minutieusement ces sujets afin de poser, dans un deuxième moment, des problèmes constitutifs transcendantaux concernant leur donation phénoménale. Dans le texte Logique et théorie générale de la science, de 1917-8, Husserl précise comment doit être cette description pré-phénoménologique des thèmes culturels. L'auteur souligne comme un des domaines matériels qui exigent une enquête ontologique propre celui de « l'esprit commun » ${ }^{2}$, c'est-àdire, de la vie culturelle en communauté. Selon Husserl, « il s'ouvre aussi ici un champ de considérations a priori, une ontologie de l'esprit commun, une doctrine eidétique a priori qui précède toutes les sciences empiriques de l'esprit, tout comme l'a priori de la nature précède les sciences naturelles $»^{3}$. Il est ici proposé de façon explicite le développement d'une ontologie de l'esprit, c'est-à-dire, d'une discipline qui explicite les traits définitionnels des relations sociales et de la production culturelle prises comme un domaine matériel spécifique, irréductible (dans ses légalités a priori) à ceux de la conscience et de la nature. Comme nous le verrons par la suite, ce sera la principale route par laquelle Husserl abordera les thèmes relatifs à la culture dans bon nombre de ses textes : on cherche à décrire des caractéristiques générales qui permettent de distinguer les phénomènes culturels en tant qu'instances d'une région eidétique matérielle relativement autonome.

\section{Ce qu'est la culture}

Afin d'esquisser la portée de l'exploration ontologique de la culture dans l'œuvre de Husserl, nous allons nous référer aux cinq articles préparés pour la

\footnotetext{
${ }^{1}$ Que les relations culturelles se fondent dans les actes des individus insérés dans la nature mondaine $n$ 'est pas sans conséquences pour les déterminations culturelles ellesmêmes. Par exemple, les individus sont soumis aux mêmes lois naturelles autour le monde, bien que ressignifiées selon les prédicats culturels en vigueur ; cela offre un sol commun au moins pour la reconnaissance de faits universellement valides, ce qui contribue au consensus interculturel. Sur ce point, voir Staiti, A. «Different worlds and tendency to concordance. Towards a new perspective on Husserl's phenomenology of culture », The New Yearbook for Phenomenology and Phenomenological Philosophy, vol X, 2010, p. 127-143 ; voir aussi Benoist, J. «'Le monde pour tous' : universalité et 'Lebenswelt' chez le dernier Husserl », Recherches husserliennes, vol.5, 1996, p. 27-52.

${ }^{2}$ Husserl, E. Logik und allgemeine Wissenschaftstheorie. Vorlesungen 1917/18. Hua XXX. Dordrecht : Kluwer, 1995, § 64, p. 283.

${ }^{3}$ Ibid.
} 
revue Kaizo entre les années 1923-1924, et publiés ensemble, seulement de manière posthume, sous le titre générique « Sur le Renouveau » ${ }^{1}$. Une des préoccupations centrales de Husserl dans ces textes est le rôle de l'éthique dans les transformations de la vie culturelle. Mais avant même de détailler ce rôle, l'auteur note que les possibilités d'action dans les contextes sociaux sont délimitées par les contraintes structurelles des interactions humaines elles-mêmes. On entrevoit, à ce moment, des recherches qui explicitent des déterminations intrinsèques de l'agir individuel et social, afin que les personnes profitent au mieux des ressources disponibles, évitent des erreurs typiques, etc. : « Seule la science rigoureuse peut procurer ici des méthodes sûres et des résultats fixes ; elle seule peut donc fournir le travail théorique préalable dont dépend une réforme rationnelle de la culture $»^{2}$. De cette façon, Husserl renvoie les questionnements éthiques à une problématique théorique fondatrice, celle de circonscrire les délimitations structurelles auxquelles toute action éthique est soumise. Il ne nous importe pas dans cet article de qualifier ce que serait l'agir éthique et quel serait son rôle dans le renouveau des formes culturelles actuelles, mais plutôt de mettre en évidence quel type de connaissance pourrait même offrir un fondement rationnel pour les relations pratiques entre les personnes. Il s'agit précisément de cette connaissance eidétique ou ontologique, citée dans la section précédente. Dans un extrait du quatrième article, cette proposition est formulée de façon encore plus claire : «La science rigoureuse [...] a aussi la tâche fondatrice d'étudier toutes les opérations s'accomplissant dans l'efficacité personnelle, singulière et communautaire, d'après leurs genres et leurs formes fondamentales, et de déterminer leurs principes normatifs comme leurs figures normatives et, par là, de traiter des domaines culturels possibles et de leurs formes normales $\rangle^{3}$. Ici s'annonce la tâche de systématiser les structures

\footnotetext{
${ }^{1}$ Husserl, E. Sur le Renouveau. Cinq articles. Trad. L. Joumier. Paris : Vrin, 2005 (désormais cité R). Pour une reprise générale du sens de ces articles, cf. Welton, D. "Husserl and the Japanese", in Moran, D.; Embree, L. (eds.). Phenomenology : Critical Concepts in Philosophy. New York: Routledge, 2004, p. 313-340; Yu, C.-C. "Husserl on ethical renewal and philosophical rationality : intercultural reflection", Investigationes fenomenológicas, n. 9, 2012, p. 145-156.

${ }^{2}$ R, p. 25.

${ }^{3}$ R, p. 75.
} 
invariantes de la vie culturelle sous ses formes diverses, c'est-à-dire, une ontologie matérielle tournée vers des restrictions eidétiques qui délimitent un type d'être, à savoir, celui des relations spirituelles ou culturelles.

Husserl admet que la connaissance eidétique du domaine spirituel n'a pas encore été développée ${ }^{1}$, mais il est possible de reconnaître, dans quelques passages des articles sur le Renouveau, des tentatives de les ébaucher. Dans le troisième texte (intitulé «Renouveau en tant que problème éthique-individuel »), Husserl concentre les principaux thèmes de sa recherche eidétique sur la culture. Revenons sur ces thèmes à partir de cette citation un peu longue :

Une humanité s'étend aussi loin que l'unité d'une culture ; au degré supérieur, d'une culture universelle close autonome qui peut comprendre en elle de nombreuses cultures nationales particulières. Dans une culture s'objective précisément une unité de vie active dont le sujet d'ensemble est l'humanité correspondante. Par culture nous n'entendons en effet rien d'autre que l'ensemble des opérations qui ont lieu dans les activités suivies d'hommes communautarisés et qui ont leur existence spirituelle durable dans l'unité de la conscience communautaire et de sa tradition qui se maintient. Sur le fondement de leur incarnation physique, de leur expression, qui les aliène du créateur originaire, elles sont, dans leur sens spirituel, objets possibles d'expérience pour tous ceux qui sont à même de les comprendre après-coup. Elles peuvent, au cours du temps, toujours redevenir des points de rayonnement d'effets spirituels portés sur des générations toujours nouvelles dans le cadre de la continuité historique. Et c'est précisément en cela que tout ce que comprend le titre de culture a son genre essentiel propre d'existence objective et fonctionne d'autre part comme une source continuelle de la communautarisation ${ }^{2}$.

Husserl offre ici de manière très concentrée les principales lignes de recherche de la science eidétique de l'esprit. Au début, est attestée une apparente tension entre deux phénomènes constitutifs de «l'esprit commun ». D'un côté, la culture paraît être coextensive de ce que Husserl appelle « humanité » (l'ensemble des personnes réparties parmi des nations les plus diverses). L'auteur suggère même l'élargissement à la limite de cette coextensivité, de sorte qu'il possible au moins concevoir une culture universelle correspondant à l'unification des diverses humanités particulières. D'un autre côté, il convient de noter que

${ }^{1}$ « Or, de l'autre côté, nous avons bien des sciences nombreuses et fécondes rapportées au domaine de l'esprit ou encore de l'humanité, mais ce sont de part en part des sciences empiriques, et «simplement» empiriques. (...) Ici manque précisément la science apriorique parallèle, pour ainsi dire la mathesis de l'esprit et de l'humanité » (R., p. 26-27).

${ }^{2}$ R, p. 42 . 
chaque humanité (partielle) produit effectivement des formes culturelles particulières avec lesquelles elle finit par s'identifier. Ainsi, la diversité humaine est liée à une multiplication des cultures. Le fait que la culture soit coextensive à l'humanité n'implique pas qu'il s'agisse toujours des mêmes formes culturelles qui s'imposeraient comme des blocs inéluctables sur les personnes insérées dans les communautés les plus diverses. L'humanité se laisse reconnaître par l'unité culturelle, mais sans que cette unité soit celle d'une simple soumission à un ensemble unique de formes interactionnelles partagées par tous.

À ce stade, au moins deux fronts de recherche se font jour : d'une part, recenser les types d'unités culturelles déjà particularisés ; de l'autre, rendre visibles les invariants ultimes des formes culturelles, de façon à établir les déterminations eidétiques permettant de reconnaître la culture en général. Husserl développe les deux types de recherche; par exemple, dans le cinquième article sur le Renouveau, l'auteur cherche à délimiter « un premier type $»^{1}$ de culture, centré sur des normes d'empreinte religieuse, ayant en vue un contraste avec la culture scientifique, un thème exploré par Husserl dans de nombreux textes. Par ailleurs, les célèbres analyses sur la crise de l'humanité européenne cherchent à attirer l'attention sur la dissolution des formes culturelles qui auraient pour noyau la pensée rationnelle. Nous reviendrons brièvement sur ce thème à la fin de notre exposé. Ce qui nous intéresse maintenant est de formuler les invariants qui délimitent la notion même de culture en général, un thème qui nous semble moins abordé par les commentateurs de l'œuvre husserlienne.

Revenons à la longue citation mentionnée plus haut. La culture y est présentée comme l'ensemble de productions de sens des personnes en communauté, productions qui deviennent accessibles pour ceux qui partagent les moyens expressifs dans lesquels ces sens sont véhiculés, en les reproduisant conformément aux normes de compréhension en vigueur au sein de la communauté. En d'autres termes, la culture dérive des personnes rassemblées en une communauté, comme production qui est transmise aux membres (avec un degré d'adhésion plus ou moins grand) et qui collabore au maintien des liens communautaires eux-mêmes. On constate une remarquable circularité dans l'analyse husserlienne de la culture : d'une certaine manière, la culture résulte des interactions sociales au moyen desquelles les personnes établissent des liens communautaires et justement apprennent à se reconnaitre comme personnes ; mais les sens culturels sont constamment transmis à d'autres personnes et même à de nouvelles générations, et de cette façon apparaissent, d'après les mots de Husserl, " comme une source constante de communautarisation ». Il n'existe donc pas quelque chose comme « la culture » qui pourrait

${ }^{1}$ R, p. 82 . 
être isolée des interactions sociales, puisque c'est dans la réalisation des interactions sociales que les formes culturelles s'instituent et deviennent une dimension instituante de la société.

Ces constatations nous mènent à reconnaître une distinction analytique centrale pour comprendre la complexité intrinsèque à la région matérielle « esprit », à savoir, celle entre socialité et culture. Considérons quelques aspects de la première. La socialité est elle-même fondée par des structures invariantes bien spécifiques, lesquelles découlent d' « actes sociaux ». Husserl commente ce point : «La communauté est une subjectivité personnelle, pour ainsi dire à plusieurs têtes et pourtant liée. Ses personnes singulières sont ses «membres", entrelacés les uns aux autres fonctionnellement à travers des « actes sociaux » aux multiples figures, unissant spirituellement la personne à la personne (actes je-tu; ordres, conventions, activités amoureuses, etc.) $»^{1}$. Analysées du point de vue de l'ontologie de l'esprit, les sociétés se révèlent instituées par des paramètres normatifs d'interaction. Les «actes sociaux» sont ceux dans lesquels les individus agissent de façon complémentaire ou réciproque, accomplissant des fonctions partielles qui, correctement articulées, produisent un sens mutuellement partagé. Cela apparaît clairement, par exemple, dans l'exercice de la communication. En apprenant à communiquer, le sujet normalise ses capacités intentionnelles en conformité avec les paramètres d'expression socialement partagés. Dans le texte «Phénoménologie de la communauté de communication », Husserl reconnaît que « le fondement de la socialité appartient à une activité à plusieurs niveaux », qui enveloppe depuis l'attestation perceptive de l'autre sujet jusqu'à l'instauration effective d'une interaction sociale, qui se produirait « quand en parlant [avec autrui] je manifeste mon intention et je le motive, dans notre situation commune, à comprendre ce que je dis, et que je me tourne vers lui $»^{2}$. Communiquer suppose donc la coordination des actes individuels en vue de l'élaboration d'un sens partageable. L'agent communicatif n'est pas le sujet enfermé dans ses capacités intrasubjectives, mais le «nous », c'est-à-dire, les sujets agents dont les actes partiels se réfèrent intrinsèquement aux actes des autres sujets afin de compléter son sens. Cet élément notable de la communication est à la base de tout acte social/culturel : le sujet ne se limite pas à satisfaire ses intentions isolées, mais il se soumet à une normativité supra-individuelle. De cette manière, les communautés ont pour condition a priori un système d'actes sociaux qui obligent

\footnotetext{
${ }^{1}$ R, p. 42.

${ }^{2}$ Husserl, E. Zur Phänomenologie der Intersubjektivität. Texte aus dem Nachlass. Dritter Teil. 1929-35. Hua XV. Den Haag : Martinus Nijhoff, 1973, p. 475.
} 
les individus à soumettre leurs capacités isolées à des rôles normalisés qui se complètent et forment ainsi des relations mutuellement compréhensibles ${ }^{1}$.

Notons à présent qu'outre cette structuration intrinsèque des actes des individus en tant qu'ils sont soumis à la réciprocité, la socialité renvoie aussi à des aspects culturels comme conditions a priori. Dans un extrait du quatrième article sur le renouveau, Husserl suggère ce point : " Les formes de la vie, de l'efficacité, de la configuration culturelle dans laquelle une communauté est concrète et, dans son ensemble, a sa configuration formelle typique déterminée, doivent avoir la plus grande signification de valeur» ${ }^{2}$. Husserl associe ici les configurations culturelles et l'existence concrète de la société ellemême. La culture attribue de la détermination aux possibilités d'interactions sociales. Considérés en tant que tels dans leur structuration a priori, les actes sociaux sont des systèmes d'obligation mutuelle qui attribuent des rôles complémentaires aux individus, lesquels se socialisent en soumettant leurs capacités individuelles aux normes de comportements fonctionnels, c'est-à-dire, liés aux fonctions sociales qui doivent être exercées mutuellement. Mais que ces interactions soient, par exemple, de tels ou tels types, ou impliquées dans telles hiérarchies, qu'elles aient tels ou tels systèmes d'interdictions et de mérite, tout cela dépend de la concrétisation des formes sociales. Cette délimitation des formes concrètes des systèmes sociaux, cette sélection, cristallisation et reproduction de certaines fonctions sociales privilégiées, d'où résultent des institutions avec certaines relations hiérarchiques, des systèmes spécifiques de parenté, etc., est l'exercice de la culture, ou, en d'autres termes, c'est la culture en tant qu'exercice concret de la socialité. Alors, loin d'être un système symbolique fermé et autonome, la culture est le processus propre de concrétisation de la société, dans lequel les relations privilégiées, les coutumes promues, etc., se sédimentent en structures reproductibles pour les individus qui alors se reconnaissent comme membres de cette société.

C'est là une contribution importante de Husserl à la réflexion anthropologique contemporaine autour de la culture. De nombreuses variantes de la théorie sociale distinguent trois groupes thématiques à explorer : premièrement, les individus dans leur capacité intentionnelle d'action et de compréhension; ensuite, les relations sociales et les institutions entendues conformément aux formes typiques d'interaction et, enfin, la culture entendue comme une sorte de système symbolique relativement autonome, même s'il imprègne les

\footnotetext{
${ }^{1}$ Nous avons analysé les composants eidétiques de la socialité dans Sacrini, M. «O espírito objetivo segundo Husserl », Phainomenon, n. 27, 2018, p. 23-52.

${ }^{2}$ R, p. 67-8.
} 
niveaux précédents ${ }^{1}$. Bien qu'il soit légitime de discriminer théoriquement ces trois ordres thématiques, il serait artificiel de les traiter comme des entités isolées. Dans ses textes de l'ontologie sociale, Husserl ébauche un modèle fécond pour comprendre les relations de dépendance mutuelle entre ces ordres. Il est évident que les individus sont à la base de toute action sociale, de sorte que sans la participation active des personnes, il n'y aurait pas quelque chose comme « la société ». Du point de vue husserlien, cela ne signifie pas, cependant, que les relations et les institutions sociales soient réductibles aux actes individuels. En fait, comme déjà suggéré, les relations sociales supposent une normativité propre, irréductible aux simples paramètres intrasubjectifs pour l'exercice des capacités intentionnelles ${ }^{2}$. Il y a, en ce sens, une circularité entre les individus en tant que base intentionnelle pour les relations sociales et ces dernières en tant qu'elles délimitent les attentes normatives auxquels les individus eux-mêmes doivent se soumettre afin d'utiliser les ressources sociales disponibles (langages, outils, institutions, etc.) au moyen desquels ils pourront s'exprimer et établir des projets personnels. À son tour, comme déjà suggéré plus haut, Husserl souligne une autre circularité, entre cette normativité intersubjective liée aux rôles sociaux (et qui constitue l'essence de la socialité) et les configurations culturelles. La socialisation est le fruit des interactions constantes, dont les formes générales peuvent être explicitées dans leur pureté eidétique comme des conditions ultimes de tout acte social. À leur tour, les façons dont ces possibilités structurelles de socialisation se précisent dans les interactions particulières délimitent la culture. Cela signifie que la culture n'est pas seulement le résultat des interactions sociales, puisqu'elle est la sédimentation et la reproduction de formes spécifiques d'interactions. Les paramètres de la socialisation sont transmis intergénérationnellement, de manière que leur caractère culturel deviennent une condition pour maintenir l'unité sociale de la communauté. En d'autres mots, les rôles sociaux conférés par les systèmes fonctionnels de réciprocité coordonnent les situations dans lesquelles les individus apprennent à développer socialement leur personnalité et leurs capacités

\footnotetext{
${ }^{1}$ Ce schéma tripartite renvoie au moins jusqu'à E. Durkheim (Cf. Durkheim. E. De la division du travail social. Paris : Puf, 2007 [1893]. ; Durkheim, E. Les règles de la méthode sociologique. Paris : Payot, 2009 [1894]).

${ }^{2}$ Par exemple, pour émettre des sons, il suffit que l'individu commande correctement son corps, dans le cas où il dispose des capacités psychosomatiques nécessaires ; or, pour s'impliquer dans un dialogue, l'individu doit soumettre sa capacité à produire des sons aux attentes normatives contenues dans une situation d'interaction, de façon que ses actes particuliers exercent des rôles partiels qui seront complétées par les rôles des autres personnes : parler dans une langue compréhensible, alterner sa parole avec la parole des autres, donner des réponses pertinentes, etc.
} 
intentionnelles. Et l'exercice de ces schémas fonctionnels génère nécessairement des spécificités culturelles (certains styles d'interaction privilégiés, d'usage des matériels disponibles, d'exercice des hiérarchies, etc.) qui se sédimentent et sont transmis comme des schémas de socialisation. La culture est, de ce point de vue, un moyen de structuration de l'unité sociale, et ses configurations rendent les personnes aptes à se socialiser, concrétisant les rôles sociaux au-delà du simple squelette formel de relations réciproques concevables.

Comme on peut le voir, selon la conception husserlienne, les trois groupes thématiques de la théorie sociale ne sont pas abordés comme des étants imperméables, mais comme des parties abstraites d'un processus de dépendance mutuelle : les individus instituent les actes sociaux à partir de leurs capacités subjectives, mais en les soumettant justement à des normes intersubjectives qui sont formatrices de leur propre personnalité. À son tour, l'exercice des rôles sociaux se produit toujours sous une certaine spécificité culturelle, à l'intérieur de laquelle les interactions sociales (et même la formation subjective qui en découle) se concrétisent. Par conséquent, ce n'est pas que la culture soit une chose finie que l'individu doit simplement assimiler ; dans les processus d'interactions constantes, par lesquels le sujet apprend à se comporter comme acteur social, les modes culturelles sont instituées, transformées et transmises. La culture se laisse appréhender, conformément à la recherche eidétique de la région " esprit », bien plus comme un exercice qui concrétise les formes de socialisation plutôt que comme un système symbolique statique présupposé dans tout acte social.

\section{Les contenus spécifiquement culturels}

On a vu que la région de l'esprit peut être analysée, en termes eidétiques, en deux types de déterminations a priori, celles relatives aux pures formes de socialisation et celles relatives aux pures formes de concrétisation culturelle des interactions sociales. Considérons ce dernier facteur. De quelle manière les relations sociales s'exercent-elles en tant que relations chargées culturellement ? Cette question renvoie à la distinction entre prédicats réels et prédicats de sens, élaborée par Husserl, par exemple, dans le cours Nature et esprit, de 1919. L'auteur y explique que les choses mondaines attestées empiriquement portent des « prédicats réels " ${ }^{1}$, liés à leur constitution naturelle sous les contraintes spatiales, temporelles et causales en vigueur. Ces prédicats réels sont

\footnotetext{
${ }^{1}$ Husserl, E. Natur und Geist. Vorlesungen Sommersemester 1919. HuaM IV. Dordrecht : Kluwer, 2002, p. 125, dorénavant cité comme NG.
} 
attestables conformément à l'exercice des capacités perceptives, bien qu'ils ne se réfèrent, en tant que tels, à aucune activité subjective comme leur source d'être. En contraste avec de tels prédicats constitutifs des choses mondaines, Husserl délimite les «prédicats de signification» ou « de sens », lesquels se réfèrent à «l'opération d'actes subjectifs » ${ }^{1}$ en tant que leur origine. Il est question ici, par exemple, des caractéristiques de beauté, d'utilité, de finalité, et qui peuvent être, pour ainsi dire, intégrées à des objets réels. Cette attribution de sens aux objets matériels ne suppose pas quelconque transformation de ces dernières. Un arbre de forme saillante peut être pris pour un signe sur un che$\min ^{2}$. La propriété « être signe pour » n'altère en rien la réalité de l'arbre, qui continue d'exister conformément à les restrictions spatiotemporelles-causales qui déterminent sans cesse son être naturel. Simplement, une nouvelle couche thématique se configure, dont les caractéristiques dérivent d'actes intentionnels, qui attribuent la propriété d' «être signe » à l'arbre. Cet exemple permet de voir avec clarté que les propriétés de sens sont d'un autre ordre que celles qui sont réelles, bien que les premières dépendent des secondes pour être exprimées concrètement ${ }^{3}$.

Dans un autre extrait du cours, Husserl cherche à détailler la spécificité des prédicats de sens. L'auteur affirme :

Tous les prédicats des simples objets naturels se rapportent essentiellement à l'extension, mais les prédicats de signification ne le font que de manière impropre, à savoir, lorsqu'ils ont un objet naturel comme substrat. Un drapeau a de l'extension et des prédicats extensionnels en tant qu'objet naturel ; les prédicats de signification, qui composent le sens « drapeau » sont irréels; en soi, ils n'ont aucune relation essentielle à l'espace 4 .

L'exemple de l'arbre comme signe prenait une attribution inessentielle de sens à un objet naturel ; à présent, en mentionnant le drapeau, il en ressort un élément forgé délibérément pour exprimer un contenu significatif (la reconnaissance d'une certaine nation), lequel est véhiculé au moyen d'un certain arrangement des propriétés réelles de l'objet « drapeau » (certaines couleurs et dessins sur un morceau de tissu aux dimensions standardisées). Même dans le cas de l'objet produit délibérément, Husserl insiste sur le fait qu'il ne s'agit pas d'ajouter de nouvelles propriétés réelles à l'objet, même s'il y a manifestement

\footnotetext{
${ }^{1} \mathrm{NG}$, p. 122.

${ }^{2}$ Cf. NG, p. 124.

${ }^{3}$ On entrevoit sur ce point un aspect eidétique central de la culture : l'objectivation des prédicats de sens. Nous reviendrons sur ce thème dans la prochaine partie.

${ }^{4} \mathrm{NG}$, p. 128.
} 
une manipulation du matériel selon des modèles décidés préalablement. Les propriétés réelles du drapeau sont celles qui renvoient aux composants physiques. Que ce tissu coloré représente telle nation est une propriété d'un autre ordre, non pas directement dérivé des caractéristiques qui soutiennent la matérialité du drapeau, mais des actes subjectifs qui ont attribué tel sens à tel chose. Le caractère « indiquer la nation $X$ » n'est pas un élément réel nouveau, mais plutôt une caractéristique irréelle, un composant de sens exprimé par le support matériel, sans s'identifier à celui-ci. Les matériaux naturels peuvent être modifiés de façon à mieux « corporaliser » le contenu de sens qui y est véhiculé ; mais celui-ci reste une production subjective, susceptible d'être exprimée à travers différents supports matériels, sans qu'aucun d'eux n'épuise ou ne « réalise » de façon exclusive le sens en vue ${ }^{1}$. Par exemple, des ustensiles sont produits en série, et chaque exemplaire instancie le « même » sens qui y est incorporé, lequel ne s'identifie à aucune de ses instances ${ }^{2}$.

Cette relative autonomie des prédicats de sens permet de les prendre comme marque de la culture elle-même . Husserl formule le « concept le plus large de culture $»^{3}$ comme englobant toute objectité dotée de prédicats de sens. La culture se laisse alors circonscrire, de ce point de vue, comme le domaine des contenus significatifs qui, à leur origine, renvoient à la production subjective (par contraste avec les prédicats réels, qui ne sont qu'attestés subjectivement), et qui s'incorporent de façons différentes dans des supports matériels réels ${ }^{4}$. Examinons de plus près quelles sont les principales formes d'élaboration des prédicats de sens.

\section{Les formes eidétiques d'élaboration de la culture}

Nous avons vu que la culture n'est pas un système autonome, mais un composant du processus de socialisation auquel les individus, en tant qu'ils font partie de la région ontologique " esprit commun », sont constamment soumis. En ce qui concerne ce point, Husserl en arrive à parler de «culture en sens étroit

\footnotetext{
${ }^{1}$ Sauf, bien entendu, les objets dont la matérialité utilisée pour l'expression du contenu qui y est en vigueur est considérée comme partie intégrante du sens global présentée, comme c'est le cas des œuvres d'art.

${ }^{2}$ Husserl applique aux objets culturels un schéma similaire à celui par lequel il comprend l'instanciation des sens par le langage. Nous reviendrons sur ce thème dans la prochaine partie.

${ }^{3} \mathrm{NG}$, p. 138

${ }^{4}$ « La culture serait, donc, le corrélat de la subjectivité opérant» (NG, p. 139).
} 
comme culture sociale $»^{1}$. Nous avons vu également que les éléments spécifiquement culturels d'un tel processus sont les prédicats de signification, qui, en dernière instance, renvoient à des capacités productives subjectives. Cela serait le sens le plus large de culture, cité plus haut. Les deux sens ne sont pas séparés, bien sûr. La culture dans le sens plus général (la production subjective) s'effectue dans des situations culturelles sociales. Husserl se préoccupe également de mettre en relief les principales formes par lesquelles cette socialisation de prédicats culturels opère effectivement. Sur ce point, le long extrait de l'article sur le renouveau offre les clés de l'analyse. Deux formes eidétiques de l'établissement des relations culturelles étaient indiqués dans ce texte.

En premier lieu, la culture implique l'objectivation de l' « unité de vie active » en vigueur dans la communauté. Loin de se réduire à un ensemble de représentations mentales, les configurations culturelles se fixent de façon accessible aux membres de la communauté. Husserl accentue dans cet extrait cité, ainsi que dans son cours de Nature et esprit, l'objectivation des prédicats de sens comme condition de leur transmissibilité sociale. Il incombe de rechercher quels sont les principaux types d'objectivations des configurations culturelles et leur rôle dans la transmission des contenus thématiques qui marquent une certaine unité communautaire. Husserl paraît en distinguer au moins trois types :

a) l'objectivation des significations via le langage : au moins depuis $R e$ cherches Logiques, Husserl prend les expressions linguistiques comme des instances de significations idéelles. De ce point de vue, chaque énonciation particulière situe spatiotemporellement un sens en lui-même irréel qui ne s'y épuise pas, puisqu'il pourrait être reproduit de nouvelles fois dans de différentes énonciations particulières ${ }^{2}$. Dans des textes plus tardifs, Husserl souligne que cette instanciation des sens irréels concrétise des contenus partageables en interactions sociales, de façon à rendre clair que l'exercice communautaire du langage est une des formes privilégiées de production et dissémination de la culture. Le célèbre manuscrit sur l'origine de la géométrie est exemplaire à propos de cette thématique ${ }^{3}$. Husserl y caractérise trois moments, intimement liés entre eux, pour la disponibilisation des sens idéels relatifs au

\footnotetext{
${ }^{1} \mathrm{NG}$, p. 139.

${ }^{2}$ Cf. Husserl, E. Logische Untersuchungen. Zweiter Teil. Untersuchungen zur Phänomenologie und Theorie der Erkenntnis. Hua XIX/1. Den Haag: Martinus Nijhoff, 1984, première recherche, $\S 11$.

${ }^{3}$ Husserl, E. Die Krisis der europäischen Wissenschaften und die transzendentale Phänomenologie. Hua VI. Den Haag : Martinus Nijhoff, 1976, p. 365-386, dorénavant cité comme K.
} 
champ de la géométrie. Le premier moment est celui de la « configuration intrapsychiquement constituée $»^{1}$, c'est-à-dire, de la reconnaissance originaire du thème en vue, dont la validité est attestée. Husserl accentue l'expérience de l'évidence comme celle dans laquelle le caractère idéellement valide du thème géométrique en question est compris à l'origine : quelqu'un comprend pour la première fois que certaine relation géométrique porte une validité universelle. Cette évidence originaire est un évènement psychologique éphémère, qui deviendra très vite une expérience passée. Or, le sens idéel révélé dans sa validité peut être remémoré à des moments ultérieurs comme le même, de façon que l'évidence initiale ne s'identifie pas à un moment précis, mais peut être réactivée dans de nouvelles expériences ${ }^{2}$. Jusqu'ici, cependant, le domaine de l'expérience vécue individuelle n'est pas dépassé et, comme l'affirme Husserl, " aucune 'objectivité' n'est encore donnée ${ }^{3}$. Une seconde étape doit ensuite se dérouler, celle de la "compréhension » linguistique réciproque ${ }^{4}$, c'est-àdire, du partage intersubjectif de l'évidence perçue individuellement. Il est question ici de la transmission du sens via des actes significatifs adressés à des interlocuteurs intéressés. Par la communication, le sens devient un bien commun, réitéré par divers interlocuteurs capables de reconnaître la validité de ce qui auparavant n'était valable que pour un individu. Husserl mentionne encore un troisième moment de la stabilisation des contenus de sens, à savoir, l'écriture, capable d'attribuer une " existence durable $»^{5}$ aux sens transmis oralement. La communication orale dépend des situations concrètes dans lesquelles les interlocuteurs interagissent effectivement. Déjà, l'inscription matérielle du sens protège le contenu des variations d'intérêt et d'attention des membres de la communauté, en garantissant sa permanence comme un bien disponible virtuellement pour de nouvelles générations qui sachent déchiffrer les codes des registres employés. Le sens s'objective comme quelque chose qui ne dépend pas d'un locuteur déterminé et qui, à son tour, s'ouvre à d'innombrables interlocuteurs. La documentation ou l'inscription graphique attribue, de cette manière, une sédimentation sensible au contenu de sens, le rendant un bien durable au cours des générations.

\footnotetext{
${ }^{1} \mathrm{~K}, 370$.

2 « Intervient nécessairement, avec la remémoration active du passé, une activité concomitante de production effective, et surgit alors un « recouvrement » originaire, l'évidence de l'identité : ce qui est maintenant originairement effectif est le même que l'antérieurement évident » $(\mathrm{K}, \mathrm{p} .370)$.

${ }^{3}$ K, p. 370 .

${ }^{4} \mathrm{~K}$, p. 371.

${ }^{5}$ Ibid.
} 
b) l'objectivation des moyens de répondre aux nécessités corporelles : le fait que les personnes soient des étants psychophysiques impose des restrictions décisives au déroulement de la culture en général. Husserl présente la quotidienneté des personnes comme ordonnée autour de nécessités très basiques (alimentation, sommeil, abri, excrétion, etc. ${ }^{1}$. La satisfaction de ces carences oblige les sujets à entrer en relation avec leur milieu en tant que foyer des ressources pour leur propre conservation. Et l'établissement de modes typiques de satisfaction institue des formes culturelles spécifiques. En particulier, la culture laisse son empreinte dans les résultats des transformations environnementales. Une culture urbanistique au sens large s'instaure, c'est-àdire, l'emploi finalisé du milieu disponible en tant que territoire socialement habitable. Dans le texte 34 du volume XXXIX de la collection Husserliana, Husserl accentue cette connexion : «Le territoire et le vivre tourné vers des fins qui lui appartiennent, qui est déterminé par les besoins humains originaires, le vivre instinctif, le vivre tourné vers des fins, en tant que vivre de la satisfaction périodique de ces besoins qui se révèlent et doivent être assouvis périodiquement ${ }^{2} \gg$. La périodicité des besoins de l'exister entraîne la modification de l'environnement, qui n'est plus simplement une localité naturelle indifférente, et devient un lieu humanisé, dans lequel la communauté rencontre les conditions matérielles pour assurer son autoconservation ${ }^{3}$. Dans la territorialité humanisée, l'espace naturel est investi de sens qui renvoient à des actions humaines visant à la satisfaction de besoins et à l'obtention de fins désirables. De cette façon, « la spatiotemporalité a pour les personnalités à tous les niveaux son sens personnel particulier (...). Ces [sens] s'objectivent dans le monde environnant personnel $»^{4}$. L'environnement transformé conformément aux intentions et finalités humaines rend objective une couche de sens qui s'institue sur les aspects matériels mondains. Les différents types de constructions architecturales, les sentiers, l'ordonnancement des espaces de convivialité ; dans tous ces composantes matériels de la vie sociale se révèlent des formes privilégiées de faire face significativement aux besoins de la vie.

\footnotetext{
${ }^{1}$ Cf. Husserl, E. Die Lebenswelt. Auslegungen der vorgegebenen Welt und ihrer Konstitution. Texte aus dem Nachlass (1916-1937). Hua XXXIX. New York: Springer, 2008, texte 51 (dorénavant cité comme L).

${ }^{2}$ L, p. 330.

${ }^{3}$ Husserl admet que le territoire humanisé ne nécessite pas d'être pris comme « une partie fixe de la terre » (L, p. 394), une fois qu'il y a des communautés nomades. Même dans ces cas, une communauté reste une "unité dans sa tradition de changement» (ibid.), laissant les marques de son mode de vie dans les différents lieux qu'elle habite. ${ }^{4}$ L, p. 395.
} 
c) l'objectivation de fonctions et finalités dans les outils : la production et l'emploi de divers types d'objets caractérisent des modes de vie culturellement spécifiques. Les matériaux naturels sont, dans cette production, «spiritualisés », c'est-à-dire, deviennent des substrats pour la stabilisation des fonctions qui répondent aux intérêts humains les plus variés. Comme Husserl affirme dans Psychologie phénoménologique, «les choses, également les choses sans vie, lorsque nous les prenons telles que nous en faisons l'expérience, peuvent être en soi le support d'une spiritualisation qu'elles peuvent avoir d'une certaine manière incorporée en elles ${ }^{1}$. De façon plus simple, même les choses naturelles peuvent être intégrées dans les pratiques quotidiennes, à mesure que ses caractéristiques permettent un usage finaliste ponctuel (reprenons l'exemple de l'arbre saillant qui devient un signe sur un sentier). En outre, il est important de mettre l'accent sur les objets qui furent délibérément planifiés selon des intérêts et des besoins, et dont les possibilités d'utilisation furent activement façonnées pour atteindre les objectifs qui leur sont associés ${ }^{2}$. Cette corporalisation des sens dans la constitution même des artefacts et des outils implique des projections d'usage qui doivent être comprises et appliquées par ceux qui apprennent les manières privilégiées de se comporter dans une certaine communauté. Selon Husserl, un objet culturel « porte en lui une signification finaliste, un pour-ça, un être déterminé et approprié pour ça $»^{3}$. En d'autres termes, l'objet spiritualisé a, inscrite dans la matérialité, une certaine prescription d'usage ; ses formes suggèrent certains types de comportement, lesquels sont renforcés communautairement selon que l'ensemble des objets est partagé. Cela veut dire que le sens culturel déposé dans le matériau naturel ne repose pas en lui-même, mais signifie justement parce qu'il renvoie à un système pratique de la communauté productrice 4 . "Les objets ont dans leurs caractères pratiques un contexte pratique propre, une synthèse de formation téléologique et, en plus, une signification totale référée à la téléologie $»^{5}$, insiste Husserl. De cette façon, chaque objet culturel exprime des intérêts et des fins valorisés socialement et offre dans sa matérialité des occasions pour la dextérité comportementale requise afin d'obtenir la fin désirée. Par exemple, la veste doit être correctement portée pour éviter le froid ; les couverts doivent

\footnotetext{
${ }^{1}$ Husserl, E. Phänomenologische Psychologie. Vorlesungen Sommersemester. 1925. Hua IX. Den Haag : Martinus Nijhoff, 1968, p. 111 (dorénavant cité comme PP).

${ }^{2}$ Cf. PP, p. 409.

${ }^{3}$ PP, p. 407.

${ }^{4}$ Sur ce thème, cf. Walton, R. "The worldhood of the world and the worldly character of objects in Husserl", in Nenon, Th., Blosser, P. (eds). Advancing Phenomenology. Essays in honor of Lester Embree. New York : Springer, 2010, p. 139-156. ${ }^{5}$ PP, p. 409.
} 
être utilisés correctement pour éviter tout contact manuel avec les aliments préparés, etc. A chaque emploi optimal des fonctionnalités inscrites matériellement dans l'objet culturel correspond un système de comportement par lequel s'accomplissent les fins visées. L'habilité à l'emploi des objets implique la reproduction de certaines pratiques corporelles culturellement privilégiées pour l'obtention de fins et la satisfaction d'intérêts socialement valorisés.

En second lieu, notons que les prédicats de sens fixés par différents types d'objectivation (linguistique, mais aussi environnemental et instrumental) doivent être intersubjectivement transmis. Les sens culturellement chargés sont liés à des objectités matérielles (au sens large, qui englobe même les mots en tant qu'unités sensibles), et il est nécessaire que le sujet appréhende ces objectités selon certaines manières privilégiées afin que le contenu en question soit pleinement disponible. En d'autres termes, les sens façonnés par la culture ne vont pas surgir dans la vie subjective comme par maturation intrinsèque ; ils doivent être assimilés dans des interactions d'apprentissage spécifiques ${ }^{1}$. La continuité intergénérationnelle des sens fixés objectivement apparaît ici en tant que marque de l'unité culturelle. En fin de compte, la validité des sens culturels ne se limite pas aux interactions présentielles momentanées. La transmissibilité s'étend entre générations, de sorte que les sens survivent à la finitude de leurs auteurs quand ils sont assumés par de nouvelles générations. Cet aspect renvoie à l'historicité intrinsèque à la vie humaine. Husserl commente ce point par exemple dans l'appendice XXVII de La Crise des sciences : "La vie humaine est en général nécessairement historique et cela avec une prégnance particulière en tant que vie culturelle $»^{2}$. Le passage du temps s'imprègne, dans le tissu des relations sociales, comme historicité culturelle, étant donné que ces relations sont enseignées et, ainsi, au moins une partie des sens qui délimitent l'environnement culturel familier est légué de façon intergénérationnelle. Du point de vue de cette inévitable historicité des prédicats de sens, liée à la finitude même des générations humaines, il en découle que la culture est vécue comme un ensemble de traditions. Le processus de socialisation par des con-

\footnotetext{
${ }^{1}$ Husserl considère que toute expérience ordonnée ne dépend pas de la transmissibilité culturelle. Dans les Méditations cartésiennes, par exemple, l'auteur cherche à circonscrire ce qui configurerait la sphère de propriété de l'égo, c'est-à-dire, ce qui serait éprouvé sans la contribution des résultats intentionnels dérivés des interactions avec les autres, et soutient qu'une division minime entre l'activité noétique et l'apparaître mondanisé s'imposerait intrinsèquement à la vie subjective. Cf. Husserl, E. Cartesianische Meditationen und Pariser Vorträge. Hua I. Den Haag : Martinus Nijhoff, 1973, $\S \S 46-47$.

${ }^{2}$ K, p. 507.
} 
figurations culturelles particularisantes institue, de cette manière, la reproductibilité intergénérationnelle des modes de vie, dont l'unité ne se fait pas uniquement par les relations directes entre les personnes, mais remonte à une origine ou une fondation antérieure. La tradition, à son tour, est un ensemble de sens qui demeure disponible au cours de l'historicité intergénérationnelle. Se démarquent ici les rôles des ancêtres et des successeurs, c'est-à-dire, de ceux qui fondent les sens transmis et de ceux qui les accueilleront dans les temps à venir. La constitution actuelle de l'environnement culturel familier n'est donc pas quelque chose d'isolé, mais renvoie au moins en partie à l'héritage reçu et n'ignore pas que les sens en vigueur maintenant seront légués. Husserl reconnaît : « N'importe quelle espèce de configuration culturelle a son historicité, a son caractère d'émergence, sa référence au futur et, de la même manière, à son humanité historiquement vivante, productrice et utilisatrice $»^{1}$. Comme nous l'avons vu, la culture dérive de la concrétion même des relations sociales spécifiques, qui favorisent l'institution de modes de compréhension et d'action familiers pour un groupe qui partage les situations interactives dans lesquelles des contenus de sens sont thématisés. Il faut à présent ajouter : les interactions n'ont pas lieu de façon aléatoire, mais dépendent des sens disponibles, c'està-dire, de ceux qui ont survécu au passage intergénérationnel et qui deviennent un sol partagé avec les générations suivantes.

Husserl cherche à discerner les principales formes que ce passage intergénérationnel des sens culturels peut assumer. La plus basique d'entre elles, et qui est liée à la possibilité même de transmission des contenus spécifiques, est la continuité, c'est-à-dire la préservation de certains sens même sous la succession des générations. Cette continuité de la transmission n'exclut pas, pour sa part, la transformation des sens accueillis par les successeurs. Les sens traditionnels sont réappropriés et ressignifiés selon, par exemple, le perfectionnement des moyens techniques en vue de l'obtention des fins considérées comme ayant de la valeur par la communauté. Dans ces cas, il est possible que les sens originaux soient occultés ; ils peuvent être, pour ainsi dire, enterrés par des appropriations biaisées et qui se satisfont de constater l'adéquation des outils disponibles aux fins souhaitées, sans la compréhension correspondant des complexités significatives inhérentes au processus en vue. Par ailleurs, ceci est la principale critique qu'adresse Husserl à la technicisation des procédures scientifiques, au sens où la pratique scientifique se réduit de nombreuses fois à l'application d'algorithmes préétablis ayant en vue l'obtention de résultats ponctuels, sans la clarté requise quant aux raisons historiques pour lesquelles

${ }^{1}$ K, p. 504. 
ces procédures-là sont choisies et ces résultats obtenus ${ }^{1}$. Selon Husserl, « la science doit nous rendre libres, d'abord libres théoriquement et ensuite libres dans tout notre agir et notre création. Mais la science qui se spécialise et se technicise ne nous rend pas même libres théoriquement $»^{2}$. Husserl en vient même à parler de décadence des pratiques culturelles à cause de la perte de leur sens originaire. Dans une large mesure, c'est sous la marque de la décadence culturelle que Husserl établit le diagnostic de la crise des sciences européennes. De façon très concise, notons que, pour Husserl, la forme spirituelle ou culturelle européenne aurait été forgée autour d'un objectif qui expliciterait quelque chose d'essentiel à l'humanité, à savoir, la possibilité de régir l'existence finie par des idéaux théoriques dont la réalisation exigerait un déroulement historique interminable. Ce résultat serait intimement lié à l'idéal de scientificité, omnienglobant et absolument fondée, jeté pour la première fois dans l'histoire par les philosophes grecs et assumé successivement par les générations qui constituèrent la forme de vie européenne. La crise remonterait aux difficultés de compréhension et de réactivation du sens de cet idéal fondateur de l'unité culturelle européenne par les générations contemporaines ${ }^{3}$. Néanmoins, la décadence n'est pas une destinée inéluctable des configurations culturelles dont le sens hérité fut recouvert par des appropriations négligentes au cours de l'enchaînement des générations. Il y a la possibilité d'un renouveau du sens, ce qui exige d'assumer activement l'historicité originaire parfois oubliée de la configuration culturelle en question, de manière à réactiver les aspects significatifs recouvert et même à les reformuler sous des critères reconnus communautairement comme valides.

\section{Considérations finales}

Nous avons cherché à ordonner différents thèmes liés à la culture dans l'œuvre de Husserl. Sans doute, serait-il nécessaire d'explorer en profondeur chacun d'entre eux. Cependant, nous avons montré l'unité d'une problématique de fond, à savoir, ébaucher les déterminations eidétiques d'un genre suprême d'être matériel, l'esprit commun. D'après notre lecture, on distingue deux

\footnotetext{
${ }^{1}$ Cf. NG, p. 6.

${ }^{2} \mathrm{NG}, p .12$.

${ }^{3}$ Sur tout ce point, voir la célèbre conférence dispensée par Husserl en 1935, La crise de l'humanité européenne et la philosophie (K, 314-348). Nous avons examiné en détail de l'analyse husserlienne de la scientificité dans Sacrini, M. A cientificidade na fenomenologia de Husserl. São Paulo : Loyola Ed., 2018.
} 
strates dans cette région ontologique : celle de la socialité, relative aux formes a priori des interactions, formes responsables de régler de façon supra-individuelle les capacités individuelles, et celle de la culture, relative à la concrétion même de la socialité au moyen des prédicats de sens. En tant qu'ensemble de délimitations eidétiques, la culture renvoie toujours à des contenus de sens/significatifs autour desquels (ou au moyen desquels) les interactions sociales se réalisent, contenus qui s'objectivent soit par le langage, soit par les transformations environnementales, ou bien encore par un réseau complexe d'outils et d'ustensiles avec lesquels les personnes interagissent quotidiennement. Les prédicats de sens sont transmis ou répandus dans ses interactions présentielles et légués de façon intergénérationnelle en tant qu'inscription matérielle qui survit à la finitude de la génération initiale et se laisse réactiver dans les expériences des générations suivantes. En des termes plus généraux, celles-ci sont les limites a priori de tout phénomène qui se laisse reconnaître comme culturel.

Bien que les analyses sur la culturalité aient un intérêt théorique propre, rappelons-nous que Husserl recherchait dans les analyses ontologiques principalement des voies de passage à la problématique phénoménologique. Nous rechercherons, en une autre étude, dans quelle mesure l'a priori culturel pose des problèmes spécifiques à l'investigation transcendantale ${ }^{1}$.

\footnotetext{
${ }^{1}$ Steinbock suggère que l'explicitation des contenus de sens en tant que thèmes transmis de façon intergénérationnelle oblige Husserl à étendre l'analyse constitutive audelà des méthodes statique et génétique et à ébaucher une méthode phénoménologique générative (Steinbock, A. Home and beyond. Generative phenomenology after Husserl. Evanston : Northwestern Univ. Press, 1995). Perreau juge qu' ' il n'est pas sûr que l'on puisse vraiment qualifier la phénoménologie de 'générative' pour désigner par là une nouvelle méthode » (Perreau, L. Le monde social selon Husserl, op. cit., p. 332). De ce point de vue, dans les textes de Husserl, la générativité serait plus un thème disséqué sous les ressources de l'analyse génétique qu'une nouvelle méthode. Il nous semble que la question reste ouverte. Pour y répondre, il faudrait clarifier la spécificité transcendantale de la transmissibilité des sens objectivés, sens qui ne sont pas mûris intrasubjectivement. En d'autres mots, il faudrait réfléchir sur la spécificité des expériences dans laquelle on établit le contact avec des objectités qui portent des prédicats de sens et qui permettent ensuite d'acquérir et de développer des capacités appréhensives (situations d'apprentissage).
} 